\title{
Reconstructing curves from their Hodge classes
}

\author{
Maria Gioia Cifani ${ }^{1}$ D $\cdot$ Gian Pietro Pirola ${ }^{2} \cdot$ Enrico Schlesinger $^{3}$
}

Received: 22 April 2021 / Accepted: 7 December 2021 / Published online: 25 January 2022

(C) The Author(s) 2022

\begin{abstract}
Let $S$ be a smooth algebraic surface in $\mathbb{P}^{3}(\mathbb{C})$. Movasati and Sertöz (Rend. Circ. Mat. Palermo $2: 1-17,2020)$ associate an ideal $I_{\alpha(C)}$ to the primitive cohomology class $\alpha(C)$ of $C$ in $S$. We show that the equations of $C$ can be determined by $I_{\alpha(C)}$ under numerical conditions. We apply this result to reconstruct rational curves and arithmetically CohenMacaulay curves from their cohomology classes. On the other hand, we show that the class $\alpha(C)$ of a rational quartic curve $C$ on a smooth quartic surface $S$ is not even perfect, that is, that $I_{\alpha(C)}$ is bigger than the sum of the Jacobian ideal of $S$ and of the homogeneous ideals of curves $D$ in $S$ for which $I_{\alpha(D)}=I_{\alpha(C)}$.
\end{abstract}

Keywords Algebraic cycles · Smooth surfaces · Arithmetically Cohen-Macaulay curves and rational curves

Mathematics Subject Classification $14 \mathrm{C} 25 \cdot 14 \mathrm{H} 50$

\section{Introduction}

The Hodge conjecture, one of the most challenging and interesting open questions in algebraic geometry, can be regarded as a weaker version of a reconstruction problem. Even when the Hodge conjecture is known, as for curves on surfaces, there are a series of somewhat related problems that might shed a new light on some aspects of the cycle map.

A good example is given by [1, Theorem 4.b.26] where it is proven that, given a smooth surface $S \subset \mathbb{P}^{3}$ and an integral class $\gamma$ in $H^{1}\left(\Omega_{S}^{1}\right)$ with the same numerical

Maria Gioia Cifani

mariagioia.cifani@uniroma3.it

Gian Pietro Pirola

gianpietro.pirola@unipv.it

Enrico Schlesinger

enrico.schlesinger@polimi.it

1 Dipartimento di matematica e fisica, Università di Roma 3, Largo San Leonardo Murialdo, 1, 00146 Roma, Italy

2 Department of Mathematics 'F. Casorati', University of Pavia, via Ferrata 5, 27100 Pavia, Italy

3 Dipartimento di Matematica 'F. Brioschi', Politecnico di Milano, Piazza Leonardo da Vinci 32, 20133 Milano, Italy 
properties as the fundamental class of a curve $C \subset S$, then $\gamma$ is itself the fundamental class of an effective divisor $D \subset S$ provided $\operatorname{deg}(S)$ is large relative to the self-intersection of $\gamma$ and to $\operatorname{deg}(C)$.

In a similar vein, very interesting recent work by Movasati and Sertöz [2] concerns the reconstruction of subvarieties in hypersurfaces of $\mathbb{P}^{N}$ from their periods. Our purpose is to give an answer, in the special case of curves lying on a smooth algebraic surface $S$ in complex projective space, to two questions raised in [2] that we now illustrate. A curve $C$ in $S$ has a fundamental cohomology class $\eta_{C} \in H^{1}\left(\Omega_{S}^{1}\right)$. We denote by $\alpha(C)$ the equivalence class of $\eta_{C}$ in the quotient of $H^{1}\left(\Omega_{S}^{1}\right)$ modulo the subspace generated by the class $\eta_{H}$ of a plane section of $S$ : the class $\alpha(C)$ depends on the embedding of $S$ in $\mathbb{P}^{3}$, and can be seen as a linear form on the primitive cohomology $H^{1}\left(\Omega_{S}^{1}\right)^{\perp_{H}}$. Following [2] we focus our analysis on the annihilator $I_{\alpha(C)}$ of $\alpha(C)$ in the polynomial ring $R=H_{*}^{0}\left(\mathcal{O}_{\mathbb{P}^{3}}\right)$. Note-see Proposition 2.3 - that $I_{\alpha(C)}=I_{\alpha(D)}$ for two curves $C$ and $D$ in $S$ if and only if $m C+n D+p H$ is linearly equivalent to zero for some choice of integers $m, n$ and $p$ with $m$ and $n$ non zero and relatively prime. Thus the annihilator $I_{\alpha(C)}$, which contains the homogeneous ideal $I_{C}$ of $C$ and the Jacobian ideal $J_{S}$ of $S$, in general it is much larger than $I_{C}+J_{S}$, as it contains the ideal $I_{D}$ for any curve $D$ for which there is a relation $m C+n D+p H \sim 0$ as above. Still, one can ask whether $C$ can be reconstructed from $I_{\alpha(C)}$ when $\operatorname{deg}(S)$ is large with respect to the degree or other invariants of $C$, and Movasati and Sertöz in [2] investigate, in a more general context than ours, the following questions:

(1) Under which conditions $I_{\alpha(C)}$ reconstructs $C$, in the sense that forms of low degree in $I_{\alpha(C)}$ cut out the curve $C$ scheme-theoretically? To be precise, we will say that $C$ is reconstructed at level $m$ by $I_{\alpha(C)}$ if its homogeneous ideal $I_{C}$ is generated over $R$ by $I_{\alpha(C), \leq m}$ - that is, by forms of degree $\leq m$ in $I_{\alpha(C)}$.

(2) As the example of complete intersection suggests, they define a class $\alpha \in H^{1}\left(\Omega_{S}^{1}\right) / \mathbb{C} \eta_{H}$ to be perfect at level $m$ if there exist effective divisors $D_{1}, \ldots, D_{q}$ in $S$ such that $I_{\alpha\left(D_{i}\right)}=I_{\alpha}$ for every $i=1, \ldots q$ and

$$
I_{\alpha, j}=\sum_{i=1}^{q} I_{D_{i}, j}+J_{S, j} \quad \text { for every } j \leq m
$$

where $J_{S}$ denotes the Jacobian ideal of $S$. The question is under which conditions the class $\alpha(C)$ is perfect at level $m$, and whether all classes $\alpha(C)$ are perfect at every level $m$.

In this paper we prove two theorems that give partial answers to these questions. Our first theorem extends known results on complete intersections [2,3] to arithmetically CohenMacaulay curves (ACM curves for short). The tools we need for this are provided by a very nice paper by Ellingsrud and Peskine [4] which unfortunately seems to be little known. In [4] the authors were interested in the study of the Noether-Lefschetz locus, and the invariant $\alpha(C)$ plays a prominent role in their work because it vanishes if and only if the curve is a complete intersection of $S$ and another surface. Their paper connects the class $\alpha(C)$ to the normal sequence arising from the inclusions $C \subset S \subset \mathbb{P}^{3}$ and gives an effective tool for computing its annihilator $I_{\alpha(C)}$-see Lemma 2.5. To state our first theorem, given a curve $C$ in $\mathbb{P}^{3}$, we let $s(C)$ be the minimum degree of a surface containing $C$, and $e(C)$ the index of speciality of $C$, that is, the maximum $n$ such that $\mathcal{O}_{C}(n)$ is special, that is, $\left.h^{1}\left(\mathcal{O}_{C}(n)\right)\right)>0$.

Theorem 1.1 Suppose $C$ is an ACM curve on the smooth surface $S \subseteq \mathbb{P}^{3}(\mathbb{C})$. Let s denote the degree of $S$. Then 
(1) if $s \geq 2 e(C)+8-s(C)$, the curve $C$ is reconstructed at level e $(C)+3$ by $I_{\alpha(C)}$;

(2) The class $\alpha(C)$ is perfect at level $m$ for every $m$.

For example, let $C$ be a twisted cubic curve: $C$ is then ACM with invariants $s(C)=2$ and $e(C)=-1$. By Theorem 1.1, if $S$ is a quartic surface containing $C$, then $C$ is cut out scheme-theoretically by the quadrics whose equations lie in $I_{\alpha(C, S)}$. This was suggested and verified for thousands of randomly chosen quartic surfaces containing $C$ in [2, Sects. 2.3 and 3.2].

Our second theorem provides a first example of a non-perfect algebraic class $\alpha(C)$, giving a negative answer to Question 2.12 in [2].

Theorem 1.2 Let $C \subset \mathbb{P}^{3}$ be a smooth rational curve of degree 4 contained in a smooth surface $S$ of degree $s=4$. The class $\alpha(C)$ in $S$ is not perfect at level 3.

It would be very interesting to determine conditions for a class $\alpha(C)$ to be perfect, and we don't know whether ACM curves form the largest set of curves $C$ whose classes $\alpha(C)$, in any smooth surface $S$ containing $C$, are perfect.

Finally, we note that Movasati and Sertöz pose their questions of reconstruction and perfectness in a more general context, namely for classes in $H^{n}\left(\Omega_{X}^{n}\right)$ of varieties of dimension $n$ in smooth hypersurfaces $X$ in $\mathbb{P}^{2 n+1}$. An interesting and challenging problem is trying to answer those question for every $n$, generalizing as far as it is possible the results of this paper to higher dimension.

The paper is structured as follows. In Sect. 2 we collect some well known facts we need, and, for the benefit of the reader, we recall in some detail the constructions from [4] we will need in the sequel of the paper. In Sect. 3 we prove Proposition 3.1-a numerical criterion that guarantees, when the degree of $S$ is large to respect to that of $C$, that the curve $C$ is reconstructed at a certain level $m$ by $I_{\alpha(C)}$. As an example we prove in Corollary 3.2 a reconstruction result for a general rational curve of degree $d$. In Sect. 4 we prove Theorem 1.1, which is split in Theorems 4.1 and 4.8. In Sect. 5 we prove Theorem 1.2.

\section{Preliminaries}

We work in the projective space $\mathbb{P}^{3}$ over the field $\mathbb{C}$ of complex numbers. Given a coherent sheaf $\mathscr{F}$ on $\mathbb{P}^{3}$ and $i \in \mathbb{N}$, we define

$$
H_{*}^{i}(\mathcal{F})=\bigoplus_{n \in \mathbb{N}} H^{i}\left(\mathbb{P}^{3}, \mathcal{F}(n)\right) .
$$

These are graded module over the polynomial ring

$$
R=H_{*}^{0}\left(\mathcal{O}_{\mathbb{P}^{3}}\right) \cong \mathbb{C}[x, y, z, w] .
$$

Given a subscheme $X$ of $\mathbb{P}^{3}$, we will denote by $\mathscr{I}_{X}$ its sheaf of ideals, and by $I_{X}=H_{*}^{0}\left(\mathscr{I}_{X}\right)$ its saturated homogeneous ideal in $R$. We will write $I_{X, n}$ to denote its $n^{\text {th }}$ graded piece $H^{0}\left(\mathbb{P}^{3}, \mathscr{I}_{X}(n)\right)$.

If $M=\bigoplus_{n \in \mathbb{Z}} M_{n}$ a graded $R$-module, the graded $\mathbb{C}$-dual module $M^{*}$ of $M$ is defined by setting $\left(M^{*}\right)_{m}=\operatorname{Hom}_{\mathbb{C}}\left(M_{-m}, \mathbb{C}\right)$ with multiplication $R_{n} \times\left(M^{*}\right)_{m} \rightarrow\left(M^{*}\right)_{m+n}$ defined by 


$$
g \lambda(v)=\lambda(g v), \quad \forall g \in R_{n}, \lambda \in\left(M^{*}\right)_{m}, v \in M_{-m-n} .
$$

By Serre's duality, if $X \subseteq \mathbb{P}^{N}$ is an equidimensional Cohen-Macaulay subscheme of dimension $d$, then for any locally free sheaf $\mathcal{F}$ on $X$ there is an isomorphism of graded $R$-modules

$$
\left(H_{*}^{i}(X, \mathcal{F})\right)^{*} \cong H_{*}^{d-i}\left(X, \mathcal{F}^{\vee} \otimes \omega_{X}\right)
$$

Let $S$ be a smooth algebraic surface of degree $s$ in $\mathbb{P}^{3}$, and $C \subset S$ a curve, that is, an effective Cartier divisor in $S$. The curve $C$ has a cohomology class $\eta_{C} \in H^{1}\left(S, \Omega_{S}^{1}\right)$. It can be defined as follows: the curve $C$ defines a linear form $\lambda_{C}$ on the set of $(1,1)$ forms by integration; abstractly one can define this linear form as the image of the trace map $H^{1}\left(C, \Omega_{C}^{1}\right) \rightarrow \mathbb{C}$ under the transpose of the morphism $H^{1}\left(S, \Omega_{S}^{1}\right) \rightarrow H^{1}\left(C, \Omega_{C}^{1}\right)$ obtained by restricting differentials on $S$ to $C$ [5, Chapter III Ex. 7.4]. The cohomology class $\eta_{C}$ is the image of $\lambda_{C}$ under the Serre's duality isomorphism $H^{1}\left(\Omega_{S}^{1}\right)^{*} \cong H^{1}\left(\left(\Omega_{S}^{1}\right)^{\vee} \otimes \omega_{S}\right)=H^{1}\left(\Omega_{S}^{1}\right)$ : note that $\Omega_{S}^{1}$ is a rank two vector bundle with determinant $\omega_{S}$, hence we can identify $\left(\Omega_{S}^{1}\right)^{\vee} \otimes \omega_{S}$ with $\Omega_{S}^{1}$.

If $\mathcal{O}_{S}(C)$ denotes the invertible sheaf on $S$ corresponding to $C$, then $\eta_{C}=c\left(\mathcal{O}_{S}(C)\right)$ where $c$ denotes the first Chern class homomorphism

$$
c: \operatorname{Pic}(S) \rightarrow H^{1}\left(S, \Omega_{S}^{1}\right) .
$$

The perfect pairing $\langle, \quad\rangle$ of Serre's duality is compatible with the intersection product of divisor classes [5, Chapter V Ex. 1.8] in the sense that for every pair of Cartier divisors $D$ and $E$ on $S$

$$
\left\langle c\left(\mathcal{O}_{S}(D)\right), c\left(\mathcal{O}_{S}(E)\right)\right\rangle=D \cdot E .
$$

Since $S$ is a surface in $\mathbb{P}^{3}$, numerically equivalent divisors on $S$ are linearly equivalent, and the first Chern class map Pic $(S) \rightarrow H^{1}\left(S, \Omega_{S}^{1}\right)$ is injective.

The cotangent bundles of $S$ and $\mathbb{P}^{3}$ are related by the exact sequence

$$
0 \rightarrow \mathcal{O}_{S}(-s) \rightarrow \Omega_{\mathbb{P}^{3}}^{1} \otimes \mathcal{O}_{S} \rightarrow \Omega_{S}^{1} \rightarrow 0 .
$$

It is well known (see e.g. [6]) that $H^{1}\left(\Omega_{\mathbb{p}^{3}}^{1} \otimes \mathcal{O}_{S}\right) \cong \mathbb{C}$ and that its image in $H^{1}\left(\Omega_{S}^{1}\right)$ is the class $\eta_{H}$ of a plane section $H$ of $S$. We look at a portion of the long cohomology sequence arising from (2)

$$
H^{1}\left(\Omega_{S}^{1}\right) \stackrel{\delta}{\rightarrow} H^{2}\left(\mathcal{O}_{S}(-S)\right) \stackrel{\epsilon}{\rightarrow} H^{2}\left(\Omega_{\mathbb{P}^{3}}^{1} \otimes \mathcal{O}_{S}\right)
$$

Dualizing and using Serre's duality we get an exact sequence

$$
H^{0}\left(\mathcal{T}_{\mathbb{P}^{3}}(s-4)\right) \stackrel{\epsilon^{*}}{\rightarrow} H^{0}\left(\mathcal{O}_{S}(2 s-4)\right) \rightarrow \operatorname{Im}(\delta)^{*} \rightarrow 0
$$

We denote by $J_{S}$ the Jacobian ideal of $S$, that is, the ideal of $R$ generated by the partial derivatives of an equation of $S$. Then the above discussion is summarized by Griffith's theorem: the primitive first cohomology group of $S$ is isomorphic to the $(2 s-4)$-graded piece of the Jacobian ring of $S$ :

$$
H^{1}\left(\Omega_{S}^{1}\right)^{\perp_{H}} \cong \operatorname{Im}(\delta)^{*} \cong \frac{H^{0}\left(\mathcal{O}_{\mathbb{P}^{3}}(2 s-4)\right)}{J_{S, 2 s-4}}
$$


Definition 2.1 Given a curve $C$ in $S$, we will denote by $\alpha(C)=\alpha\left(C, S, \mathbb{P}^{3}\right)$ the image of its cohomology class $\eta_{C}$ under the map

$$
H^{1}\left(\Omega_{S}^{1}\right) \stackrel{\delta}{\rightarrow} H^{2}\left(\mathcal{O}_{S}(-s)\right) \cong H^{0}\left(\mathcal{O}_{S}(2 s-4)\right)^{*}
$$

Thus $\alpha(C)$ is a linear form on $H^{0}\left(\mathcal{O}_{S}(2 s-4)\right)$ that vanishes on $J_{S, 2 s-4}$.

Given $\alpha \in H^{0}\left(\mathcal{O}_{S}(2 s-4)\right)^{*}$, we denote by $I_{\alpha}$ the annihilator of $\alpha$ in the polynomial ring $R$ : it is the homogeneous ideal in $R$ whose $n^{\text {th }}$ graded piece is

$$
I_{\alpha, n}=\left\{f \in R_{n} \mid \alpha(f g)=0, \forall g \in H^{0}\left(\mathcal{O}_{S}(2 s-4-n)\right)\right\} .
$$

Remark 2.2 When writing the paper, we decided to take all ideals in the polynomial ring $R=H_{*}^{0}\left(\mathcal{O}_{\mathbb{P}^{3}}\right)$ : thus $J_{S}$ and $I_{\alpha}$ are for us ideals of $R$, and $J_{S} \subset I_{\alpha(C)}$. Our motivation is that we would like to compare $I_{\alpha}$ with the ideal of $C$ as a curve in $\mathbb{P}^{3}$. In [2] the author's denote by $I_{\alpha}$ the annihilator of $\alpha$ in the Jacobian ring and by $\tilde{I}_{\alpha}$ its preimage in $R$.

Let $T=R / I_{S}=H_{*}^{0}\left(\mathcal{O}_{S}\right)$. Then $\alpha \in\left(T_{2 s-4}\right)^{*}$, and the ideal $I_{\alpha}$ is determined by $\operatorname{Ker}(\alpha) \subseteq T_{2 s-4}$; conversely, one can recover $\operatorname{Ker}(\alpha)$ as the image of $I_{\alpha, 2 s-4}$ via the quotient map $R_{2 s-4} \rightarrow T_{2 s-4}$. The perfect pairing

$$
R_{n} / I_{\alpha, n} \times\left(R_{2 s-4-n} / I_{\alpha, 2 s-4-n}\right)^{*} \rightarrow \mathbb{C}
$$

shows $A:=R / I_{\alpha}=\bigoplus_{n=0}^{2 s-4} A_{n}$ is an artinian Gorenstein ring of socle $2 s-4$ [4, Prop 1.3].

In [4] the authors were interested in the study of the Noether-Lefschetz locus, and the invariant $\alpha(C)$ plays a prominent role in their work because it vanishes if and only if the curve is a complete intersection of $S$ and another surface. More generally, a Lefschetz type theorem about the Picard group of $S$ (see [7-9] ) implies the following fact:

Proposition 2.3 Let $C$ and $D$ be effective divisors on a smooth surface $S \in \mathbb{P}^{3}$, and let $H$ denote a plane section of $S$. Then $I_{\alpha(C)}=I_{\alpha(D)}$ if and only if there exist $m, n, p \in \mathbb{Z}, m, n \neq 0$ and relatively prime, such that $m C+n D+p H$ is linearly equivalent to zero.

Proof Suppose $m C+n D+p H$ is linearly equivalent to zero and $m$ and $n$ are nonzero. The cotangent complex (2) gives rise to an exact sequence in cohomology

$$
H^{1}\left(\Omega_{\mathbb{P}^{3}}^{1} \otimes \mathcal{O}_{S}\right) \cong \mathbb{C} \stackrel{\gamma}{\rightarrow} H^{1}\left(\Omega_{S}^{1}\right) \stackrel{\delta}{\rightarrow} H^{2}\left(\mathcal{O}_{S}(-s)\right) \simeq H^{0}\left(\mathcal{O}_{S}(2 s-4)\right)^{*}
$$

and one knows that $\gamma(1)=\eta_{H}$, so that the kernel of $\delta$ is the $\mathbb{C}$-line spanned by $\eta_{H}$. From $m C+n D+p H \sim 0$ we then deduce $m \alpha(C)=-n \alpha(D)$. Since $m$ and $n$ are nonzero, the linear forms $\alpha(C)$ and $\alpha(D)$ have the same kernel, hence $I_{\alpha(C)}=I_{\alpha(D)}$.

In the other direction, suppose $I_{\alpha(C)}=I_{\alpha(D)}$, that is, $\alpha(C)$ and $\alpha(D)$ have the same kernel. Then $\alpha(C)=c \alpha(D)$ for a nonzero complex number $c$. Using (5) and the intersection pairing we deduce that there are integers $m, n, p$, with $m$ and $n$ nonzero, such that $m C+n D+p H$ is linearly equivalent to zero. Finally, $m$ and $n$ can be taken relatively prime because $\operatorname{Pic}(S) / \mathbb{Z} H$ has no torsion (see for example [8, Theorem B]). In particular, when $D=0$, one can take $m=1$.

As noted in [4] and [2, Lemma 2.3], the ideal $I_{\alpha(C)}$ contains the ideal of $C$ in $S$. This follows from the remark of [4] that $\alpha(C) \in H^{0}\left(\mathcal{O}_{S}(2 s-4)\right)^{*}$ is the pull-back of a linear 
form $\beta(C) \in H^{0}\left(\mathcal{O}_{C}(2 s-4)\right)^{*}$. For the benefit of the reader and for later use, we give a proof of this fact. The linear form $\beta(C)$ arises from the normal bundles exact sequence:

$$
0 \rightarrow \mathcal{N}_{C / S} \cong \omega_{C}(4-s) \rightarrow \mathcal{N}_{C / \mathbb{P}^{3}} \rightarrow \mathcal{N}_{S / \mathbb{P}^{3}} \otimes \mathcal{O}_{C} \cong \mathcal{O}_{C}(s) \rightarrow 0
$$

Tensoring (6) with $\mathcal{O}_{C}(-s)$ and taking cohomology we obtain a map $H^{0}\left(\mathcal{O}_{C}\right) \rightarrow H^{1}\left(\omega_{C}(4-2 s)\right)$ and we let

$$
\beta(C) \in H^{0}\left(\mathcal{O}_{C}(2 s-4)\right)^{*} \cong H^{1}\left(\omega_{C}(4-2 s)\right)
$$

denote the image of $1 \in H^{0}\left(\mathcal{O}_{C}\right)$.

Proposition 2.4 [4, Construction 1.8] The linear form $\alpha(C)$ is the pull-back of $\beta(C)$ to $S$, that is, $\alpha(C)=\rho^{*}(\beta(C))$ where $\rho^{*}$ is the transpose of the natural map $\rho: H^{0}\left(\mathcal{O}_{S}(2 s-4)\right) \rightarrow H^{0}\left(\mathcal{O}_{C}(2 s-4)\right)$.

Proof Observe that $\Omega_{S}^{1}$ is a rank two vector bundle with determinant $\omega_{S}$, hence the tangent bundle $\mathcal{T}_{S}=\left(\Omega_{S}^{1}\right)^{\vee}$ is isomorphic to $\Omega_{S}^{1} \otimes \omega_{S}^{-1}=\Omega_{S}^{1}(4-s)$. The tangent complex of $S \subseteq \mathbb{P}^{3}$ and the normal bundle sequence (6) give rise to a commutative diagram

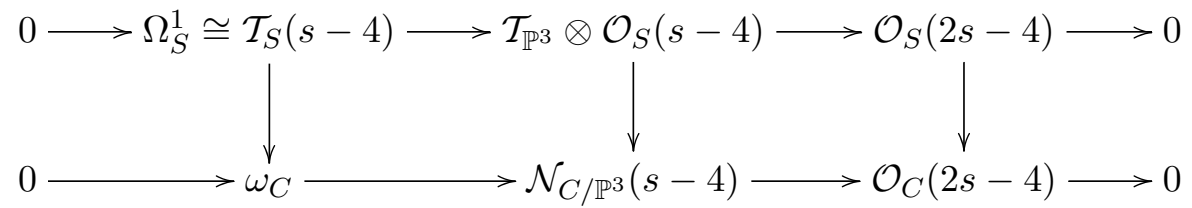

Taking cohomology and dualizing one sees that $\alpha(C)$ is the pull back of $\beta(C)$ to $S$.

The following Lemma in [4] gives an effective method to compute $I_{\alpha}$ in many cases.

Lemma $2.5\left[4\right.$, Lemma 1.10] Let $N(C)$ denote the image of the map $H_{*}^{0} \mathcal{N}_{C / \mathbb{P}^{3}}(-s) \rightarrow H_{*}^{0}\left(\mathcal{O}_{C}\right)$ arising from the normal bundle sequence (6). Let $\pi: R=H_{*}^{0}\left(\mathcal{O}_{\mathbb{P}^{3}}\right) \rightarrow H_{*}^{0}\left(\mathcal{O}_{C}\right)$ be the natural map. Then, for every integer $n$,

$$
\pi^{-1}\left(N(C)_{n}\right) \subseteq I_{\alpha(C), n}
$$

with equality if $\pi_{2 s-4-n}$ is surjective.

Proof The exact sequence

$$
H_{*}^{0} \mathcal{N}_{C / \mathbb{P}^{3}}(-s) \longrightarrow H_{*}^{0}\left(\mathcal{O}_{C}\right) \stackrel{1 \mapsto \beta}{\longrightarrow}\left(H_{*}^{0}\left(\mathcal{O}_{C}(2 s-4)\right)\right)^{*}
$$

shows $N(C)=\operatorname{Ann}_{H_{*}^{0}\left(\mathcal{O}_{C}\right)}(\beta)$.

The map $\pi: R \rightarrow H_{*}^{0}\left(\mathcal{O}_{C}\right)$ factors through $\rho: H_{*}^{0}\left(\mathcal{O}_{S}\right) \rightarrow H_{*}^{0}\left(\mathcal{O}_{C}\right)$. To simplify notation, write $T=H_{*}^{0}\left(\mathcal{O}_{S}\right)$ and $e=2 s-4$. As $\alpha$ is an element of the $T$-module $T^{*}$, the ideal $I_{\alpha}$, which by definition is the annihilator of $\alpha$ in $R$, is the inverse image of $\operatorname{Ann}_{T}(\alpha)$ under the surjective map $R \rightarrow T$. Hence what we have to prove is that $\rho^{-1}\left(N(C)_{n}\right) \subseteq \operatorname{Ann}_{T}(\alpha)_{n}$ for every integer $n$, with equality holding when $\rho_{e-n}$ is surjective. Now 


$$
\operatorname{Ann}_{T}\left(\alpha=\rho^{*}(\beta)\right)_{n}=\left\{g \in T_{n}: g \rho^{*}(\beta)(v)=\beta(\rho(g) \rho(v))=0 \quad \forall v \in T_{e-n}\right\}
$$

while the inverse image $\rho^{-1}\left(N(C)_{n}\right)$ of the $n$th graded piece of the annihilator of $\beta(C)$ in $H_{*}^{0}\left(\mathcal{O}_{C}\right)$ is equal to

$$
\left\{g \in T_{n}:(\rho(g) \beta)(w)=\beta(\rho(g) w)=0 \quad \forall w \in H^{0}\left(\mathcal{O}_{C}(e-n)\right)\right\} .
$$

The thesis is now evident.

Corollary 2.6 The annihilator $I_{\alpha(C)}$ of $\alpha(C)$ contains both the homogeneous ideal of $C$ and the Jacobian ideal of the surface $S$.

To exemplify the scope of this construction, we remark that it immediately yields the following well known corollary (originally due to Griffiths and Harris, see [6] for more details).

Corollary 2.7 Suppose $S$ is a smooth surface in $\mathbb{P}^{3}$ and $C$ is an effective divisor on $S$. Then $C$ is a complete intersection of $S$ and another surface if and only if the sequence (6) of normal bundles splits.

Proof If $C$ is a complete intersection of $S$ and another surface, it is clear that the sequence splits. Conversely,if the sequence splits, then $\beta(C)=0$. Therefore $\alpha(C)=0$, and the thesis follows from Proposition 2.3.

\section{Reconstruction of the ideal}

Motivated by [2], we want to compare $I_{C}$ and $I_{\alpha(C)}$. The following proposition gives sufficient conditions for the curve $C$ to be reconstructed at level $p$ by $I_{\alpha(C)}$. We will see that these conditions are rather sharp and useful when we consider the examples of rational curves (Corollary 3.2 and Theorem 5.1 below) and of arithmetically Cohen-Macaulay curves (Theorem 4.1)-for a specific example, if $C$ is a smooth rational quartic curve in a smooth quartic surface $S$, then $I_{\alpha(C), 2}=I_{C, 2}$ by Proposition 3.1, while the class $\alpha(C)$ in $S$ is not even perfect at level 3 by Theorem 5.1. Recall that we denote by $\mathscr{I}_{C}$ the ideal sheaf of $C$ in $\mathbb{P}^{3}$.

Proposition 3.1 Let $S$ be a smooth surface of degree $s$ in $\mathbb{P}^{3}$, and let $C$ be an effective Cartier divisor on $S$. Assume that the homogeneous ideal $I_{C}$ is generated by its forms of degree $\leq p$ and that the following vanishing conditions are satisfied

(1) $h^{1}\left(\mathscr{I}_{C}(2 s-4-p)\right)=0$

(2) $h^{0}\left(\mathcal{N}_{C / \mathbb{P}^{3}}(p-s)\right)=0$

then $I_{\alpha(C), p}=I_{C, p}$, therefore $C$ is reconstructed at level $p$ by $I_{\alpha(C)}$.

Proof Since $h^{0}\left(\mathcal{N}_{C / \mathbb{P}^{3}}(p-s)\right)=0$, the annihilator of $\beta(C)$ in degree $p$ vanishes. Since $\left.\pi_{2 s-4-p}: R_{2 s-4-p} \rightarrow H^{0} \mathcal{O}_{C}(2 s-4-p)\right)$ is surjective, by Lemma 2.5

$$
I_{\alpha(C), p}=\pi_{p}^{-1}\left(\operatorname{Ann}\left(\beta_{C}\right)_{p}\right)=I_{C, p} .
$$


We can now answer a question raised in [2, Section 2.3.1] about twisted cubics contained in quartic surfaces: if $C$ is a twisted cubic contained in a smooth quartic surface $S \subset \mathbb{P}^{3}$, then $C$ is cut out by quadrics in $I_{\alpha(C)}$. More generally:

Corollary 3.2 Suppose $C \subset \mathbb{P}^{3}$ is a general rational curve of degree $d \geq 3$ and let $n_{0}$ be the round up of $\sqrt{6 d-2}-3$, that is, the smallest positive integer $n$ such that $\left(\begin{array}{c}n+3 \\ 3\end{array}\right)-n d-1 \geq 0$. If $C$ s contained in a smooth surface $S$ of degree $s \geq n_{0}+3$, then $C$ is reconstructed at level $n_{0}+1$ by $I_{\alpha(C, S)}$.

Proof By [10] a general rational curve is a curve of maximal rank, that is, $h^{0}\left(\mathscr{I}_{C}(n)\right)=0$ for $n \leq n_{0}-1$ and $h^{1}\left(\mathscr{I}_{C}(n)\right)=0$ for $n \geq n_{0}$. Hence $C$ is $n_{0}+1$ regular in the sense of Castelnuovo-Mumford, and $I_{C}$ is generated by its forms of degree $\leq n_{0}+1$. Furthermore, by [11] the normal bundle of the immersion $\mathbb{P}^{1} \rightarrow C \subset \mathbb{P}^{3}$ is isomorphic to $\mathcal{O}_{\mathbb{P}^{1}}(2 d-1) \oplus \mathcal{O}_{\mathbb{P}^{1}}(2 d-1)$. Hence $h^{0}\left(\mathcal{N}_{C / \mathbb{P}^{3}}(-m)\right)=0$ for every $m \geq 2$. Thus we can apply Proposition 3.1 with $p=n_{0}+1$.

Remark 3.3 If $C$ is a smooth irreducible curve of degree $d$, then $h^{1}\left(\mathscr{I}_{C}(n)\right)=0$ for every $n \geq d-3-e\left(\right.$ see [12] and [13]), where $e:=e(C)=\max \left\{n \mid h^{1}(\mathcal{O}(n))>0\right\}$ is the index of speciality of $C$.

Corollary 3.4 Let $S$ be a smooth surface of degree $s$ in $\mathbb{P}^{3}$, and let $C$ be an effective Cartier divisor on $S$. Suppose $\mathscr{I}_{C}$ is r-regular in the sense of Castelnuovo-Mumford. If $s \geq 2 r+1$, then $C$ is reconstructed at level $r$ by $I_{\alpha(C)}$.

Proof Since $\mathscr{I}_{C}$ is $r$-regular, the ideal $I_{C}$ is generated by its forms of degree $\leq r$ and $H^{1}\left(\mathscr{I}_{C}(n)\right)=0$ for every $n \geq r-1$. As $s \geq 2 r+1$ and $r \geq 1$, the first condition $h^{1} \mathscr{I}_{C}(2 s-4-r)=0$ in Proposition 3.1 is satisfied for $p=r$.

We are left to check that $h^{0} \mathcal{N}_{C / \mathbb{P}^{3}}(r-s)=0$.

By [14, Prop 4.1], there are two surfaces $S_{1}$ and $S_{2}$ of degree $r$ meeting properly in a complete intersection

$$
X=S_{1} \cap S_{2}=C \cup D
$$

so that $C$ and $D$ have no common component. Consider the exact sequence

$$
0 \rightarrow \mathscr{I}_{X} \rightarrow \mathscr{I}_{C} \rightarrow \mathscr{I}_{C, X} \rightarrow 0 .
$$

Applying $\operatorname{Hom}\left(-, \mathcal{O}_{C}\right)$ we get

$$
0 \rightarrow \operatorname{Hom}\left(\mathscr{I}_{X}, \mathcal{O}_{C}\right) \rightarrow \mathcal{N}_{C} \rightarrow \mathcal{N}_{X \mid C}
$$

and $\operatorname{Hom}\left(\mathscr{I}_{X}, \mathcal{O}_{C}\right)=0$ since $C$ and $D$ have no common component. Therefore, there is an inclusion

$$
\mathcal{N}_{C} \hookrightarrow\left(\mathcal{N}_{X}\right)_{\mid C}=\mathcal{O}_{C}(r) \oplus \mathcal{O}_{C}(r)
$$

hence $h^{0} \mathcal{N}_{C}(m)=0$ for $m \leq-r-1$. In particular $h^{0} \mathcal{N}_{C}(r-s)=0$ because $s \geq 2 r+1$. 


\section{Arithmetically Cohen-Macaulay curves}

In this section we explain how Example 1.15.3 in [4] extends the result about the perfection of complete intersections to the much larger class of arithmetically CohenMacaulay curves (from now on, ACM curves). Recall that a curve $C \subset \mathbb{P}^{3}$ is called $\mathrm{ACM}$ if its homogeneous ring $R_{C}=R / I_{C}$ is Cohen-Macaulay, or, equivalently, if $C$ is locally Cohen-Macaulay of pure dimension 1 and $H_{*}^{1}\left(\mathscr{I}_{C}\right)=0$. A smooth ACM curve is what classically was referred to as a projectively normal curve. We refer the reader to [15] for a detailed study of ACM curves on a surface in $\mathbb{P}^{3}$.

If $C \subset \mathbb{P}^{3}$ is an ACM curve, then $I_{C}$ has a free graded resolution of the form

$$
0 \rightarrow E=\bigoplus_{j=0}^{r} R\left(-b_{j}\right) \stackrel{\phi}{\longrightarrow} F=\bigoplus_{i=0}^{r+1} R\left(-a_{i}\right) \rightarrow I_{C} \rightarrow 0
$$

and $I_{C}$ coincides with the ideal generated by the $r \times r$ minors of $\phi$ by the Hilbert-Burch theorem-cf. [16, Proposition II.1.1 p. 37].

Applying the functor $\operatorname{Hom}_{R}\left(\bullet, R / I_{C}\right)$ to (7) as in [17, p. 428] one obtains a long exact sequence

$$
0 \rightarrow H_{*}^{0}\left(\mathcal{N}_{C}\right) \longrightarrow \bigoplus_{i=0}^{r+1} R_{C}\left(a_{i}\right) \longrightarrow \bigoplus_{j=0}^{r} R_{C}\left(b_{j}\right) \longrightarrow H_{*}^{0}\left(\omega_{C}(4)\right) \rightarrow 0
$$

The importance of this sequence for our purposes is that it allows to compute the Hilbert function $n \mapsto h^{0}\left(\mathcal{N}_{C, \mathbb{P}^{3}}(n)\right)$ of $\mathcal{N}_{C, \mathbb{P}^{3}}$ as a function of the Hilbert function $n \mapsto h^{0}\left(\mathcal{O}_{C}(n)\right)$ of $C$; we can then compute the dimension of $\operatorname{Ann}(\beta(C))_{n}$ and of $I_{\alpha(C), n}$ in terms solely of the Hilbert function of $C$ and of the degree $s$ of $S$. To justify our assertion, one needs to observe that to compute $h^{0}\left(\mathcal{N}_{C, \mathbb{P}^{3}}(n)\right)$ out of (8) one does not need to know the numbers $a_{i}$ 's and $b_{j}$ 's, but only for each $n$ the difference

$$
\#\left\{i: a_{i}=n\right\}-\#\left\{j: b_{j}=n\right\}
$$

which depends only on the Hilbert function of $C$.

As an application of this argument, we can give for ACM curves a sharp bound for the smallest integer $n$ such that $I_{\alpha(C), n}=I_{C, n}$. For this we will not need the full Hilbert function of $C$, but just its index of speciality $e:=e(C)=\max \left\{n \mid h^{1}(\mathcal{O}(n))=h^{2}\left(\mathscr{I}_{C}(n)\right)>0\right\}$ and the minimum degree $s(C)$ of a surface containing $C: s(C)=\min \left\{n \mid h^{0}\left(\mathscr{I}_{C}(n)\right)>0\right\}$. For an ACM curve $C$, the ideal $\mathscr{I}_{C}$ is $e+3$-regular because $H_{*}^{1}\left(\mathscr{I}_{C}\right)=0$. In particular, the ideal $I_{C}$ is generated in degrees $\leq e+3$, and $s(C) \leq e+3$.

Theorem 4.1 Let $S$ be a smooth surface of degree $s$ in $\mathbb{P}^{3}$. Let $C \subset S$ be an ACM curve, let $s(C)$ be the minimum degree of a surface containing $C$ and let $e(C)$ be the index of speciality of $C$.

If $s \geq 2 e(C)+8-s(C)$ hen $I_{\alpha(C),(e+3)}=I_{C,(e+3)}$. Therefore $C$ is reconstructed at level $e+3$ by $I_{\alpha(C)}$.

Proof The statement follows from Proposition 3.1 with $p=e+3$ provided we can show that $h^{0}\left(\mathcal{N}_{C / \mathbb{P}^{3}}(e+3-s)\right)=0$. For this we use the exact sequence (8), which shows that the maximum $n$ for which $h^{0} \mathcal{N}_{C, \mathbb{P}^{3}}(n)=0$ is $n=s(C)-e(C)-5$. 
Remark 4.2 A twisted cubic curve $C$ is ACM with invariants $s(C)=2$ and $e(C)=-1$. Hence from Theorem 4.1 it follows once more that, if $C$ is contained in a smooth quartic surface $S$, then $C$ is cut out by quadrics in $I_{\alpha(C, S)}$.

Remark 4.3 Theorem 4.1 improves for ACM curves the bound of Corollary 3.4 because, since $r=e+3$, then $2 e+8-s(C)=2 r+2-s(C)$.

In [2, Sect. 2.3] [18, Ch 11], motivated by the case of complete intersections, formulate the notion of a perfect class:

Definition 4.4 Let $S$ be a smooth surface of degree $s$ in $\mathbb{P}^{3}$. A class $\alpha \in H^{1}\left(\Omega_{S}\right) / \mathbb{C} \eta_{H} \subseteq H^{0}\left(\mathcal{O}_{S}(2 s-4)\right)^{*}$ is perfect at level $m$ if there exist effective divisors $D_{1}, \ldots, D_{q}$ in $S$ such that $I_{\alpha\left(D_{i}\right)}=I_{\alpha}$ for every $i=1, \ldots, q$ and

$$
I_{\alpha, j}=\sum_{i=1}^{q} I_{D_{i}, j}+J_{S, j} \quad \text { for every } j \leq m .
$$

We say the class is perfect if $I_{\alpha}=\sum_{i=1}^{q} I_{D_{i}}+J_{S}$. We make the convention that the zero class is perfect-geometrically, this amounts to consider the empty set as a (empty) curve, and is consistent with regarding the zero divisor as an effective divisor.

Example 4.5 If $C \subset S$ is the complete intersection of two surfaces meeting properly, then $\alpha(C)$ is perfect (see [2, Ex 2.11], [4, Ex 1.15.2], [3, Prop. 2.14] ). If one does not agree that the zero class is perfect, then one needs to add the condition that $C$ is cut out by two surfaces of degrees $<s=\operatorname{deg}(S)$.

We now wish to generalize the previous example to the class of ACM curves showing that, if $C$ is ACM, then the class $\alpha(C)$ is perfect. For this we need to recall more facts from [4]. Suppose the ACM curve $C$ is contained in a smooth surface $S$ of degree $s$ and equation $f=0$. Then the polynomial $f$ can be written in the form

$$
f=\sum_{i=1}^{r+1} g_{i} h_{i}
$$

where the $h_{i}$ 's are the images of the generators of the free module $F$ in the resolution (7) of $I_{C}$, Since the $h_{i}$ 's are the signed $r \times r$ minors of $\phi$, then polynomial $f$ is the determinant of the morphism $\psi: E \oplus R(-s) \rightarrow F$ obtained adding the column $\left[g_{1}, \ldots, g_{r+1}\right]^{T}$ to the matrix of $\phi$ : in other words, $\psi$ coincides with $\phi$ on $E$, and sends $1 \in R(-s)$ to $\sum_{i=1}^{p+1} g_{i} e_{i}$, where the $e_{i}$ 's are the generators of $F$. We thus obtain a resolution of $I_{C} / I_{S}$ :

$$
0 \rightarrow E \oplus R(-s) \stackrel{\psi}{\longrightarrow} F \rightarrow I_{C} / I_{S} \rightarrow 0
$$

Since $S$ is smooth, the curve $C$ is Cartier on $S$ so that $I_{C} / I_{S}$ can locally be generated by one element. It follows that the ideal $I_{r}(\psi)$ generated by the $r \times r$ minors of $\psi$ is irrelevant, that is, its radical is the irrelevant maximal ideal $(x, y, z, w)$ of the polynomial ring $R$.

Proposition 4.6 [4, Prop. 1.16] Let $C \subset \mathbb{P}^{3}$ be an ACM curve contained in the smooth surface $S$. Suppose $I_{C}$ has the resolution (7). Then 
(1) If $\psi$ is as in exact sequence (9) the presentation of $I_{C, S}$, then

$$
I_{\alpha(C)}=I_{r}(\psi)
$$

is the ideal generated by the $r \times r$ minors of $\psi$;

(2) The $n$ th-graded piece $\operatorname{Ann}(\alpha(C))_{n}$ of the annihilator of $\alpha(C)$ in $H_{*}^{0}\left(\mathcal{O}_{S}\right)$ is the image of the natural map

$$
\bigoplus_{m \in \mathbb{Z}} H^{0} \mathcal{O}_{S}(C+(n+m) H) \otimes H^{0} \mathcal{O}_{S}(-C-m H) \longrightarrow H^{0}\left(\mathcal{O}_{S}(n)\right)
$$

Remark 4.7 Note that $\operatorname{Ann}(\alpha(C))=I_{\alpha(C)} / I_{S}$. The equality $I_{\alpha(C)}=I_{r}(\psi)$ is a non trivial fact that is not given a full proof in [4]; a complete proof can be found in [19, Proposition 4.3 and p. 382].

We can now prove that the class $\alpha(C)$ of an ACM curve in a smooth surface $S$ is perfect:

Theorem 4.8 Let $C \subset S$ be an ACM curve and let $S$ be a smooth surface. Then the class $\alpha(C)$ of $C$ in $S$ is perfect.

Proof Fix an integer $n$. By Proposition 4.6Ann $(\alpha(C))_{n}$ is the image of the natural map

$$
\bigoplus_{m \in \mathbb{Z}} H^{0} \mathcal{O}_{S}(C+(n+m) H) \otimes H^{0} \mathcal{O}_{S}(-C-m H) \longrightarrow H^{0}\left(\mathcal{O}_{S}(n)\right)
$$

Note the sum on the left hand side is finite, and consists of those $m$ for which the linear systems $|C+(m+n) H|$ and $|-C-m H|$ are both non-empty. For such an $m$ we pick a basis $g_{1}, \ldots, g_{r}$ of $H^{0} \mathcal{O}_{S}(-C-m H)$ and corresponding effective divisors $D_{k}=\left(g_{k}\right)_{0} \in|-C-m H|$. The image of

$$
H^{0} \mathcal{O}_{S}\left(C+\left(n+m_{k}\right) H\right) \otimes g_{k}
$$

in $H^{0}\left(\mathcal{O}_{S}(n)\right)$ is $H^{0} \mathscr{I}_{D_{k} / S}(n)$. (if $C \sim t H$ is a complete intersection of $S$ and another surface, taking $m_{k}=-t$ and $n=0$ we get $D_{k}$ the empty curve, and in this case $\alpha(C)=0$ is perfect by our definition). Note that $\mathbb{Q} \alpha\left(D_{k}\right)=\mathbb{Q} \alpha(C)$ by Proposition 2.3. Now letting $k$ and $m$ vary we see that $\alpha(C)$ is perfect at level $n$, for every $n$. Since $\operatorname{Ann}(\alpha(C))$ is finitely generated, we can let $n$ vary up to the maximum degree of a generator of $\operatorname{Ann}(\alpha(C))$, and recover the whole $\operatorname{Ann}(\alpha(C))$ as the sum of finitely many $I_{D_{k} / S}$ with $D_{k} \sim C+(n+m) H$ for some $m$ and $n$. Therefore $\alpha_{C}$ in $S$ is perfect.

\section{Example of a non perfect class}

Theorem 5.1 Let $C \subset \mathbb{P}^{3}$ be a smooth rational curve of degree 4 contained in a smooth surface $S$ of degree $s=4$. The class $\alpha(C)$ in $S$ is not perfect at level 3 . 
Proof A smooth rational quartic curve $C \subset \mathbb{P}^{3}$ is contained in a unique quadric surface $Q$, and $Q$ is necessarily smooth (all curves on the quadric cone are arithmetically CohenMacaulay by [5, Chapter V Ex. 2.9 ]). We may assume $C$ is a divisor of type $(3,1)$ on $Q$. The ideal sheaf of $C$ is 3-regular, hence $I_{C}$ generated by quadrics and cubics.

Suppose $C$ is contained in a smooth quartic surface $S$. Then $Q \cap S$ is the union of $C$ and an effective divisor $D_{0}$ of type $(1,3)$ on $Q$. Note that $D_{0}$ is a curve of degree 4 and arithmetic genus 0; as the divisor class of $D_{0}$ is different from that of $C$ and $C$ is irreducible, we conclude that $C$ and $D_{0}$ have no common component.

The curves $C$ and $D_{0}$ don't move in their linear system on the quartic surface $S$ : for $C$ this follows from $C^{2}=-2$, and in any case for both $D_{0}$ and $C$ one might argue that

$$
h^{0}\left(\mathcal{O}_{S}\left(D_{0}\right)\right)=h^{0}\left(\mathcal{O}_{S}(2 H-C)\right)=h^{0}\left(\mathscr{I}_{C}(2)\right)=1 .
$$

Having established the geometric set-up, we proceed to show that $I_{\alpha(C)}$ contains too many cubics for $\alpha(C)$ to be perfect at level 3. To compute the dimension of $I_{\alpha(C), 3}$, we use the fact that $R / I_{\alpha(C)}$ is a Gorenstein ring with socle in degree $2 s-4=4$, hence

$$
\operatorname{dim} I_{\alpha(C), 3}=\operatorname{dim} I_{\alpha(C), 1}+\operatorname{dim} R_{3}-\operatorname{dim} R_{1}=\operatorname{dim} I_{\alpha(C), 1}+16 \geq 16 .
$$

This estimate is good enough for us to prove the theorem, but let us show anyway that $\operatorname{dim} I_{\alpha(C), 3}=16$ : as $C$ is a divisor of type $(3,1)$ on $Q, h^{1}\left(\mathscr{I}_{C}(3)\right)=0$ hence by Lemma 2.5 $I_{\alpha(C), 1}$ is the pull back to $R_{1}$ of $N(C)_{1}$, the image of $H^{0}\left(\mathcal{N}_{C / \mathbb{P}^{3}}(-3)\right)$ in $H^{0}\left(\mathcal{O}_{C}(1)\right)$; as the normal bundle of $C$ pulls-back on $\mathbb{P}^{1}$ to $\mathcal{O}_{\mathbb{P}^{1}}(7) \oplus \mathcal{O}_{\mathbb{P}^{1}}(7)$ by [11, Proposition 6]), we conclude that $I_{\alpha(C), 1}=0$, hence $\operatorname{dim} I_{\alpha(C), 3}=16$. The same argument shows that $I_{\alpha(C), 2}=I_{C, 2}$ as well.

To check whether $I_{\alpha, 3}$ is perfect, we need to determine curves $D$ in $S$ with $I_{\alpha(D)}=I_{\alpha(C)}$ and $h^{0}\left(\mathscr{I}_{D}(3)\right) \geq 1$ so that $D$ can contribute to $I_{\alpha, 3}$. Thus suppose $D$ is such a curve. By Proposition 2.3, there exist $m, n, p \in \mathbb{Z}, m, n \neq 0$ and relatively prime, such that $p H+m C+n D$ is linearly equivalent to zero. By assumption $3 H-D$ is effective; as $C$ is not linearly equivalent to $t H$ for any $t$, neither is $D$, hence $1 \leq \operatorname{deg}(D)=D \cdot H \leq 11$. Replacing $D$ with $D^{\prime}=3 H-D$ we can even assume $D \cdot H \leq 6$.

Now consider the matrix

$$
M=\left[\begin{array}{ccc}
H^{2} & C \cdot H & H \cdot D \\
C \cdot H & C^{2} & C \cdot D \\
H \cdot D & C \cdot D & D^{2}
\end{array}\right]=\left[\begin{array}{ccc}
4 & 4 & x \\
4 & -2 & y \\
x & y & z
\end{array}\right]
$$

As $p H+m C+n D$ is linearly equivalent to zero, the vector $v=[p, m, n]^{T}$ is in the kernel of $M$. Set $x=H \cdot D, y=C \cdot D$ and $z=D^{2}$. Note that $z=D^{2}=2\left(p_{a}(D)-1\right)=2 q$ is even.

The determinant of $M$ must vanish, so

$$
x^{2}+4 x y-2 y^{2}-24 q=0
$$

From this we deduce first that $x$ and $y$ must be even, and then that 4 divides $x$. As $1 \leq x \leq 6$, we must have $x=4$. Thus $D$ is a curve of degree 4 , and either $D=C$ or $C$ is not a component of $D$, hence $y=C \cdot D \geq 0$. Assume that $D \neq C$. Writing $y=2 t$ with $t \geq 0$, we obtain the equation

$$
t^{2}-4 t+3 q-2=0
$$


Looking at the discriminant of this quadratic equation in $t$ we deduce $6-3 q$ is a perfect square, so that $q=2-3 a^{2}$ for an integer $a \geq 0$. Then solving for $t$ and imposing $t \geq 0$ we obtain $t=2+3 a$. So $H \cdot D=x=4, C \cdot D=y=4+6 a$ and $D^{2}=4-6 a^{2}$. Then solving the linear system $M v=0$ for $v=[p, m, n]^{T}$ we find $m=a n$ and $p=-(a+1) n$. Since $m$ and $n$ are relatively prime and non zero and $a \geq 0$, the only possibility is that $a=1$. Then we can take $m=n=1$ and conclude $C+D \sim 2 H$, so that $C+D$ is the complete intersection of the unique quadric $Q$ containing $C$ with $S$, and $D=D_{0}$ is the residual to $C$ in the complete intersection $Q \cap S$.

We conclude that the only curves $D$ in $S$ that are contained in a cubic surface and satisfy $I_{\alpha(D)}=I_{\alpha(C)}$ are $C$, the residual $D_{0}$ to $C$ in the complete intersection $Q \cap S$, and the effective divisors linearly equivalent to either $3 H-C$ or $3 H-D_{0}$. But observe that, if $D^{\prime} \sim 3 H-D_{0} \sim C+H$ is effective, then

$$
h^{0} \mathscr{I}_{D^{\prime}}(3)=h^{0} \mathscr{I}_{C}(2)=1 .
$$

Therefore there is a unique cubic containing $D^{\prime}$, whose equation is contained in the ideal of $D_{0}$. Similarly, if $D^{\prime \prime} \sim 3 H-C$ is effective, there is a unique cubic containing $D^{\prime \prime}$, whose equation is contained in the ideal of $C$. Hence any cubic form that belongs to the ideal of a curve $D$ on $S$ satisfying $I_{\alpha(D)}=I_{\alpha(C)}$ is in the vector space spanned by $I_{C, 3}$ and $I_{D_{0}, 3}$.

To show $\alpha(C)$ is not perfect at level 3 it is now enough to show that cubics containing either $C$ or $D_{0}$ plus the cubics in the Jacobian ideal $J_{S}$ do not span $I_{\alpha(C), 3}$.

To this end, note that cubic surfaces that contain both $C$ and $D_{0}$ are in the ideal of the complete intersection of $S$ and $Q$, and so form a vector space of dimension 4. By Grassmann's formula

$$
\operatorname{dim} I_{C, 3}+\operatorname{dim} I_{D_{0}, 3}=7+7-4=10
$$

There are four independent cubics in the Jacobian ideal, so

$$
\operatorname{dim} I_{C, 3}+\operatorname{dim} I_{D_{0}, 3}+\operatorname{dim} J_{S, 3} \leq 14<16=\operatorname{dim} I_{\alpha(C), 3}
$$

and this shows that $\alpha(C)$ in $S$ is not perfect at level 3 .

Remark 5.2 The class $\alpha(C)$ of the quartic $C$ in $S$ is perfect at level 2 by Proposition 3.1 since $h^{1}\left(\mathscr{I}_{C}(2)\right)=h^{0}\left(\mathcal{N}_{C / \mathbb{P}^{3}}(-2)\right)=0$.

Acknowledgements The authors are grateful to the referee for several valuable comments. The authors are members of GNSAGA of INdAM, and were partially supported by national MIUR funds, PRIN 2017 Moduli and Lie theory, and by MIUR: Dipartimenti di Eccellenza Program (2018-2022)—Dept. of Math. Univ. of Pavia.

Open Access This article is licensed under a Creative Commons Attribution 4.0 International License, which permits use, sharing, adaptation, distribution and reproduction in any medium or format, as long as you give appropriate credit to the original author(s) and the source, provide a link to the Creative Commons licence, and indicate if changes were made. The images or other third party material in this article are included in the article's Creative Commons licence, unless indicated otherwise in a credit line to the material. If material is not included in the article's Creative Commons licence and your intended use is not permitted by statutory regulation or exceeds the permitted use, you will need to obtain permission directly from the copyright holder. To view a copy of this licence, visit http://creativecommons.org/licenses/by/4.0/. 


\section{References}

1. Griffiths, P., Harris, J.: Infinitesimal variations of Hodge structure. II. An infinitesimal invariant of Hodge classes. Compos. Math. 50(2-3), 207-265 (1983)

2. Movasati, H., Sertöz, E.C.: On reconstructing subvarieties from their periods. Rend. Circ. Mat. Palermo 2, 1-17 (2020)

3. Dan, A.: Analytic and Algebraic Geometry. In: Noether-Lefschetz locus and a special case of the variational Hodge conjecture: using elementary techniques, pp. 107-115. Hindustan Book Agency, New Delhi (2017)

4. Ellingsrud, G., Peskine, C.: Anneau de Gorenstein associé à un fibré inversible sur une surface de l'espace et lieu de Noether-Lefschetz. In Proceedings of the Indo-French Conference on Geometry (Bombay, 1989). Hindustan Book Agency, Delhi, pp. 29-42 (1993)

5. Hartshorne, R.: Algebraic Geometry. Springer, New York, Heidelberg (1977). (Graduate Texts in Mathematics, No. 52)

6. Ellingsrud, G., Gruson, L., Peskine, C., Strømme, S.A.: On the normal bundle of curves on smooth projective surfaces. Invent. Math. 80(1), 181-184 (1985)

7. Deligne,P., Katz, N.: Groupes de monodromie en géométrie algébrique. II: Lect. Notes Math. 340 (1973). Séminaire de Géométrie Algébrique du Bois-Marie 1967-1969 (SGA 7 II)

8. Bădescu, L.: A remark on the Grothendieck-Lefschetz theorem about the Picard group. Nagoya Math. J. 71, 169-179 (1978)

9. Voisin, C.: Hodge Theory and Complex Algebraic Geometry I, Volume 76 of Cambridge Studies in Advanced Mathematics. Cambridge University Press, Cambridge, Translated from the French original by Leila Schneps (2002)

10. Hartshorne, R., Hirschowitz, A.: Courbes rationnelles et droites en position générale. Ann. Inst. Fourier (Grenoble) 35(4), 39-58 (1985)

11. Eisenbud, D., Van de Ven, A.: On the normal bundles of smooth rational space curves. Math. Ann. 256(4), 453-463 (1981)

12. Gruson, L., Peskine, C.: Space curves: complete series and speciality. In: Space Curves (Rocca di Papa, 1985), Volume 1266 of Lecture Notes in Math. Springer, Berlin, pp. 108-123 (1987)

13. Han, F.: Geometrically reducible line complexes. Math. Ann. 316(4), 819-824 (2000)

14. Peskine, C., Szpiro, L.: Liaison des variétés algébriques. I. Invent. Math. 26, 271-302 (1974)

15. Hartshorne, R., Schlesinger, E.: Gonality of a general ACM curve in $\mathbb{P}^{3}$. Pacific J. Math. 251(2), 269313 (2011)

16. Martin-Deschamps, M., Perrin, D.: Sur la classification des courbes gauches. Astérisque 184, 185 (1990). (Société Mathématique de France)

17. Ellingsrud, G.: Sur le schéma de hilbert des variétés de codimension 2 dans $P^{e}$ à cône de CohenMacaulay. Ann. Sci. École Norm. Sup. (4) 8(4), 423-431 (1975)

18. Movasati, H., Loyola, R. Villaflor: A course in Hodge theory: periods of algebraic cycles

19. Kleppe, J.O., Miró-Roig, R.M.: Ideals generated by submaximal minors. Algebra Number Theory 3(4), 367-392 (2009)

Publisher's Note Springer Nature remains neutral with regard to jurisdictional claims in published maps and institutional affiliations. 